\title{
Evaluation of Free-Stall Mattress Bedding Treatments to Reduce Mastitis Bacterial Growth
}

\author{
M. A. Kristula, ${ }^{\star 1}$ Z. Dou, ${ }^{*}$ J. D. Toth, ${ }^{\star}$ B. I. Smith, ${ }^{*}$ N. Harvey, ${ }^{*}$ and M. Sabo \\ *University of Pennsylvania, School of Veterinary Medicine, New Bolton Center, Kennett Square 19348 \\ †PO Box 43, Unionville, PA 19375
}

\begin{abstract}
Bacterial counts were compared in free-stall mattresses and teat ends exposed to 5 treatments in a factorial study design on 1 dairy farm. Mattresses in five 30cow groups were subjected to 1 of 5 bedding treatments every other day: $0.5 \mathrm{~kg}$ of hydrated limestone, $120 \mathrm{~mL}$ of commercial acidic conditioner, $1 \mathrm{~kg}$ of coal fly ash, 1 $\mathrm{kg}$ of kiln-dried wood shavings, and control (no bedding). Counts of coliforms, Klebsiella spp., Escherichia coli, and Streptococcus spp. were lowest on mattresses bedded with lime. Mattresses bedded with the commercial acidic conditioner had the next lowest counts for coliforms, Klebsiella spp., and Streptococcus spp. Wood shavings and the no-bedding control had the highest counts for coliform and Klebsiella spp. Compared with wood shavings or control, fly ash reduced the counts of coliforms, whereas for the other 3 bacterial groups, the reduction was not always significant. Streptococcus spp. counts were greatest in the control group and did not differ among the shavings and fly ash groups. Teat swab results indicated that hydrated lime was the only bedding treatment that significantly decreased the counts of both coliforms and Klebsiella spp. There were no differences in Streptococcus spp. numbers on the teats between any of the bedding treatments. Bacterial populations grew steadily on mattresses and were generally higher at 36 to $48 \mathrm{~h}$ than at 12 to $24 \mathrm{~h}$, whereas bacterial populations on teats grew rapidly by $12 \mathrm{~h}$ and then remained constant. Hydrated lime was the only treatment that significantly reduced bacterial counts on both mattresses and teat ends, but it caused some skin irritation.
\end{abstract}

Key words: bedding, bacterial count, free-stall

\section{INTRODUCTION}

Mastitis is a major health problem for dairy cows, the control and treatment of which can be costly (De-

Received August 10, 2007.

Accepted January 1, 2008.

${ }^{1}$ Corresponding author: Kristula@vet.upenn.edu
Graves and Fetrow, 1993). The pathogens causing mastitis are generally characterized as either contagious or environmental in origin (Smith and Hogan, 2001). Although substantial progress has been made in controlling contagious mastitis by implementation of teat dipping and dry cow therapy programs, there has been less progress made on the control of mastitis caused by environmental pathogens (Smith and Hogan, 2001).

The primary reservoir of environmental pathogens is the environment in which the cow is living. For example, the rate of intramammary coliform infections has been correlated to the number of bacteria on the teat end (Hogan et al., 1989); and the number of bacteria at the teat end has been correlated to the number of bacteria in the bedding (Natzke and LeClair, 1976; Hogan et al., 1989). Therefore, designing housing and management systems that reduce exposure of environmental pathogens to the teat end should result in less environmental mastitis (Smith and Hogan, 2001).

Various bedding treatments have been tested for suppressing bacterial growth and reducing mastitis occurrence, most of which involve alteration of $\mathrm{pH}$ with acidic or alkaline conditioners. For example, hydrated lime reportedly reduced bacterial counts when used with sawdust (Hogan and Smith, 1997) or with recycled manure as bedding in tie-stalls (Hogan et al., 1999). Previous research also suggested that adding fly ash to manure solids used for bedding was beneficial in reducing bacterial loads (Brazil et al., 1999). Fly ash is a byproduct of fluidized bed combustion at coal-burning power plants; many of the by-products have a high $\mathrm{pH}$ (Dou et al., 2003). When mixed with animal manures, fly ash has been shown to reduce phosphorus solubility, due primarily to its high $\mathrm{pH}$ (Dou et al., 2003). If proved to be effective in suppressing bacterial growth, fly ash would be a good choice for a bedding treatment because it is available in large quantities in many regions at low or no cost for producers. Recently, commercial acidic bedding conditioners have become available. Hogan et al. (2007) found a commercial acidic bedding conditioner to be effective in suppressing gram-negative bacteria and Streptococcus spp. over the short term in recycled organic bedding. 
Table 1. Treatments ${ }^{1}$ by stall area

\begin{tabular}{llllll}
\hline & \multicolumn{5}{c}{ Stall area } \\
\cline { 2 - 6 } Week & \multicolumn{1}{c}{ I } & \multicolumn{1}{c}{ II } & III & IV & V \\
\hline 1 & Control & CAC & Shavings & Fly ash & Lime \\
2 & CAC & Control & Lime & Shavings & Fly ash \\
3 & Shavings & Lime & Fly ash & CAC & Control \\
4 & Lime & Fly ash & CAC & Control & Shavings \\
5 & Fly ash & Shavings & Control & Lime & CAC \\
\hline
\end{tabular}

${ }^{1}$ Mattress treatments: control $=$ no bedding; CAC $=$ commercial acidic conditioner $(300 \mathrm{~mL}$ for first and $120 \mathrm{~mL}$ for subsequent applications in each cycle); shavings = $1 \mathrm{~kg}$ of kiln-dried wood shavings; fly ash = $1 \mathrm{~kg}$ of coal fly ash; lime $=0.5 \mathrm{~kg}$ of hydrated lime.

Despite the growing interest in using various bedding conditioners for controlling mastitis bacteria, data are lacking regarding the effect of hydrated lime or other bedding treatments used as the sole bedding material on free-stall mattresses. The objective of the present study was to evaluate hydrated lime, a commercial acidic bedding conditioner (CAC), fly ash, and kilndried wood shavings compared with a no-bedding control, as sole free-stall mattress treatments for suppressing mastitis-causing bacterial populations.

\section{MATERIALS AND METHODS}

The experiment was conducted in June and July 2005 at the University of Pennsylvania's Marshak Dairy, a 200-cow teaching and research facility located at New Bolton Center (Kennett Square, PA). The study featured a factorial design with 5 treatments (Table 1) including hydrated lime, CAC, coal fly ash, kiln-dried wood shavings, and a no-bedding control. Treatments were applied to a group of 30 stalls in each of the 5 stall blocks (I to V) in the dairy. In subsequent sampling periods (weeks), treatments were allocated at random over the remaining 4 stall blocks, giving 5 replications of the treatments over time. On d 1 (Monday) of each of the sampling periods, the mattresses in each of the 5 stall blocks (I to V) were swept clean with a broom, and treatments $(0.5 \mathrm{~kg}$ of hydrated lime, $300 \mathrm{~mL}$ of CAC, $1 \mathrm{~kg}$ of coal fly ash, $1 \mathrm{~kg}$ of kiln-dried wood shavings, or a no-bedding control) were applied to each of the 5 stall blocks according to the schedule outlined in Table 1; on d 3 (Wednesday) and 5 (Friday), subsequent applications were made after ordinary mattress cleaning and grooming was done by the dairy staff before milking. The CAC was applied according to manufacturer recommendations at $300 \mathrm{~mL}$ per stall on $\mathrm{d} 1$ and a $120-\mathrm{mL}$ maintenance rate on $\mathrm{d} 3$ and 5 , respectively. Each application was made by hand-applying the material to the rear $61 \mathrm{~cm}$ of the $1.83-\times 1.07-\mathrm{m}$ mattresses (Mayo mattresses; www.mayomats.com) as uniformly as possible.

\section{Sample Collection}

Sample collection was initiated on d 5 (Friday) in each of the 5 treatment cycles, with the first sampling accommodating the actual milking times beginning on Friday afternoon $(0 \mathrm{~h})$, followed by sampling at 12,24 , 36 , and $48 \mathrm{~h}$ thereafter. For each sampling and from each treatment group, mattress samples were obtained from 5 stalls and teat swab samples were taken from 5 cows. Sample collection was repeated following the same procedures in the subsequent 4 wk of the study.

To obtain mattress samples, a group of 5 representative mattresses were selected for sampling from each treatment. Stalls that were wet or had gross manure contamination were not chosen to be sampled. For the selected stalls, a $15-\times 15$-cm Plexiglas quadrant was placed in the rear $61 \mathrm{~cm}$ of the mattress and all material within the quadrant was collected. Samples at $0 \mathrm{~h}$ were obtained after the treatments had been applied to the mattresses before the cows lying on the bedding. Subsequent samples were taken from the mattresses before the cows were taken to the milking parlor and before grooming of the stalls. The samples were refrigerated at $4^{\circ} \mathrm{C}$ and plated for bacterial counts the day after all the samples were taken. Ordinary mattress cleaning and grooming was performed at the normal times by the dairy staff before milking.

To obtain teat swab samples, 5 cows were randomly selected from each treatment group during the milking process at each sampling. The external teat orifices of both the right front and left hind teat of the selected cows were sampled by swabbing the teat end in a circular pattern 3 times with a cotton swab moistened in PBS, using 1 swab per teat. Together, the 10 swabs per treatment group were placed in $40 \mathrm{~mL}$ of PBS, refrigerated at $4^{\circ} \mathrm{C}$, and plated for bacteria counts the day after the final sample collection on Sunday. Samples at $0 \mathrm{~h}$ were obtained after the postmilking teat dip had been applied for $30 \mathrm{~s}$ and teats dried with a paper towel. Subsequent samples were taken when cows entered the parlor before milking and application of the teat predip. 
A preliminary trial was conducted to determine if refrigerated storage would affect bacteria populations from teat swabs. Three teat-end swab samples obtained as described above were placed in PBS individually and stored in a refrigerator $\left(4^{\circ} \mathrm{C}\right)$. Aliquots of the samples were then plated in duplicate at $0,24,48$ and $72 \mathrm{~h}$ on MacConkey's agar (gram-negative bacteria; Difco Laboratories, Detroit, MI) and tryptic soy agar (total aerobic bacteria; Becton Dickinson, Franklin Lakes, $\mathrm{NJ}$ ); colony-forming units were counted after $24 \mathrm{~h}$ of incubation at $37^{\circ} \mathrm{C}$. Changes in bacteria populations over time were tested by repeated-measures ANOVA using PROC GLM in SAS (SAS Institute Inc., Cary, NC) with a repeated statement and time as a dependent variable. Refrigeration time did not significantly affect bacteria populations plated on MacConkey (unadjusted $P>F=0.1039)$ or tryptic soy agars $(P>F=0.1814)$.

\section{Bacteriological Methods}

Bedding Samples. Twenty grams of bedding was added to $180 \mathrm{~mL}$ of sterile buffered peptone water $(\mathrm{pH}$ 7.0) in a 500-mL plastic bag and placed on a shaker at room temperature for $30 \mathrm{~min}$. After contents had settled (2 to $3 \mathrm{~min}$ ), appropriate dilutions were plated on the surface of each of 3 media: MacConkey's agar (Difco Laboratories), cystine-lactose-electrolyte deficient (CLED) agar with Andrade indicator (Oxoid Ltd., Basingstoke, UK), and Edward's modified medium agar (Remel Products, Lenexa, KS) with 5\% plasma (Hemostat Laboratories, Dixon, CA). Six 10- $\mu$ L samples of each dilution were plated on one half plate containing each agar medium and incubated for $18 \mathrm{~h}$ at $37^{\circ} \mathrm{C}$. Bacterial groups were identified as coliforms (lactosepositive pink-red colonies on MacConkey's agar), Klebsiella spp. (extremely mucoid yellow colonies on CLED agar), Escherichia coli (bright pink semitranslucent colonies with surrounding pink halo on CLED agar), or Streptococcus spp. (total growth on Edward's modified medium agar).

Teat Samples. Containers with the teat swabs and $40 \mathrm{~mL}$ of PBS were placed on a shaker for $30 \mathrm{~min}$ at room temperature. The same bacteriologic methods described previously for the bedding samples were used for the teat samples.

\section{Data Analysis}

The original bacterial counts $(\mathrm{x})$ were transformed using the formula $\ln (\mathrm{x}+1)$ before data analysis; results were reported as the natural log-transformed data. An ANOVA was performed using JMP version 4.0 (SAS Institute Inc.). Comparisons of treatment effects were tested with least squares means contrasts, where each level of a fixed effect was compared with all other levels. For levels to be considered significantly different, the probability of a type- 1 error was less than the $\alpha$-level of rejection $(P<0.05)$ for both the $t$-statistic comparison between each pair of levels and the $F$-test of all comparisons considered in the contrast.

\section{RESULTS}

\section{Mattress Samples}

There was a significant treatment effect $(P<0.0001)$ on the mean bacterial counts for all 4 bacterial groups. Hydrated lime was the most effective treatment in suppressing bacterial growth on the mattresses, with the lowest bacterial counts for all 4 types of bacteria (Table 2 ). The commercial product CAC provided the next lowest bacterial counts and was more effective than fly ash, shavings, and the no-bedding control for coliforms, Klebsiella spp., and Streptococcus spp. Fly ash effectively reduced coliform populations compared with the no-bedding control or shavings. However, populations of Klebsiella spp. and E. coli with the fly ash treatment were similar to that of the control. The shavings and control treatments had the highest Klebsiella spp. counts. Shavings had no effect on the coliform and $E$. coli counts compared with the no-bedding control. Streptococcus spp. counts were highest for the no-bedding control (Table 2).

There were also significant differences $(P<0.0001)$ in the mean natural log-transformed bacterial populations (cfu/g of DM) over the different sampling times. As shown in Table 3, bacterial counts at $0 \mathrm{~h}$ were lowest for all 4 types of bacteria although $E$. coli and Streptococcus spp. counts at $0 \mathrm{~h}$ were not different from those at $12 \mathrm{~h}$. For all bacterial types, there was a clear trend of increasing bacterial populations as sampling time progressed to $12,24,36$, and $48 \mathrm{~h}$ (Table 3 ).

In addition, the stall block areas to which the various treatments in the factorial design were applied seemed to differ in bacterial counts of coliforms, Klebsiella spp., and Streptococcus spp. $(P<0.05)$, but not for $E$. coli. Stall areas III and V were associated with lower counts of coliforms and Klebsiella spp. (Table 4).

There were significant interactions of treatment $x$ hour for coliforms, Klebsiella spp., and Streptococcus spp. $(P<0.0002)$ and treatment $\times$ stall area for all 4 types of bacteria $(P<0.005)$. Despite the treatment $\times$ stall area interactions, the effect of treatment was still significant for all 4 bacterial populations when the variation from stall area was accounted for.

\section{Teat-End Swabs}

Hydrated lime significantly reduced coliform and Klebsiella spp. counts but not E. coli or Streptococcus 
Table 2. Sample mean (ln-transformed cfu/g of DM) and post-ANOVA groups for bacterial counts from bedding samples by treatment group over all samplings

\begin{tabular}{|c|c|c|c|c|c|c|c|c|}
\hline \multirow[b]{2}{*}{ Treatment $^{1}$} & \multicolumn{2}{|c|}{ Coliforms } & \multicolumn{2}{|c|}{ Klebsiella spp. } & \multicolumn{2}{|c|}{ Escherichia coli } & \multicolumn{2}{|c|}{ Streptococcus spp. } \\
\hline & $\begin{array}{c}\text { Mean } \\
(\mathrm{SE})\end{array}$ & $\begin{array}{c}\text { Contrast }^{2} \\
(1.03)\end{array}$ & $\begin{array}{c}\text { Mean } \\
(\mathrm{SE})\end{array}$ & $\begin{array}{c}\text { Contrast } \\
(1.26)\end{array}$ & $\begin{array}{c}\text { Mean } \\
(\mathrm{SE})\end{array}$ & $\begin{array}{c}\text { Contrast } \\
(1.95)\end{array}$ & $\begin{array}{c}\text { Mean } \\
(\mathrm{SE})\end{array}$ & $\begin{array}{c}\text { Contrast } \\
(1.20)\end{array}$ \\
\hline Lime & $\begin{array}{c}8.07 \\
(1.77)\end{array}$ & $\mathrm{a}$ & $\begin{array}{c}6.39 \\
(1.65)\end{array}$ & a & $\begin{array}{c}3.96 \\
(1.48)\end{array}$ & $\mathrm{a}$ & $\begin{array}{c}7.23 \\
(2.01)\end{array}$ & a \\
\hline Shavings & $\begin{array}{c}20.73 \\
(0.40)\end{array}$ & $\mathrm{b}$ & $\begin{array}{c}19.32 \\
(0.38)\end{array}$ & $\mathrm{b}$ & $\begin{array}{l}14.93 \\
(1.76)\end{array}$ & $\mathrm{c}$ & $\begin{array}{c}20.69 \\
(0.38)\end{array}$ & $\mathrm{b}$ \\
\hline Fly ash & $\begin{array}{c}17.56 \\
(1.20)\end{array}$ & $\mathrm{d}$ & $\begin{array}{l}16.28 \\
(1.12)\end{array}$ & $\mathrm{d}$ & $\begin{array}{l}13.44 \\
(1.74)\end{array}$ & bc & $\begin{array}{c}19.64 \\
(0.92)\end{array}$ & $\mathrm{b}$ \\
\hline CAC & $\begin{array}{l}14.81 \\
(1.96)\end{array}$ & $\mathrm{c}$ & $\begin{array}{l}11.94 \\
(1.90)\end{array}$ & $\mathrm{c}$ & $\begin{array}{c}9.83 \\
(2.12)\end{array}$ & $\mathrm{b}$ & $\begin{array}{c}15.51 \\
(2.20)\end{array}$ & $\mathrm{c}$ \\
\hline Control & $\begin{array}{l}21.56 \\
(0.48)\end{array}$ & $\mathrm{b}$ & $\begin{array}{c}17.91 \\
(1.17)\end{array}$ & bd & $\begin{array}{l}13.65 \\
(2.14)\end{array}$ & $\mathrm{bc}$ & $\begin{array}{c}23.53 \\
(0.44)\end{array}$ & $\mathrm{d}$ \\
\hline
\end{tabular}

${ }^{\mathrm{a}-\mathrm{d}}$ Means within a column with different letters differ significantly $(P<0.05)$.

${ }^{1}$ Treatments: lime $=0.5 \mathrm{~kg}$ of hydrated lime; shavings $=1 \mathrm{~kg}$ of kiln-dried wood shavings; fly ash $=1 \mathrm{~kg}$ of coal fly ash; CAC = commercial acidic conditioner $(300 \mathrm{~mL}$ for first and $120 \mathrm{~mL}$ for subsequent applications in each cycle); control = no bedding.

${ }^{2}$ Standard errors for post-ANOVA contrasts.

Table 3. Sample mean (ln-transformed cfu/g of DM) and post-ANOVA groups for bacterial counts from all bedding samples by sampling time

\begin{tabular}{|c|c|c|c|c|c|c|c|c|}
\hline \multirow[b]{2}{*}{ Sample time,${ }^{1} \mathrm{~h}$} & \multicolumn{2}{|c|}{ Coliforms } & \multicolumn{2}{|c|}{ Klebsiella spp. } & \multicolumn{2}{|c|}{ Escherichia coli } & \multicolumn{2}{|c|}{ Streptococcus spp. } \\
\hline & $\begin{array}{c}\text { Mean } \\
(\mathrm{SE})\end{array}$ & $\begin{array}{c}\text { Contrast }^{2} \\
(1.03)\end{array}$ & $\begin{array}{c}\text { Mean } \\
(\mathrm{SE})\end{array}$ & $\begin{array}{c}\text { Contrast } \\
(1.26)\end{array}$ & $\begin{array}{c}\text { Mean } \\
\text { (SE) }\end{array}$ & $\begin{array}{c}\text { Contrast } \\
(1.95)\end{array}$ & $\begin{array}{c}\text { Mean } \\
(\mathrm{SE})\end{array}$ & $\begin{array}{c}\text { Contrast } \\
(1.20)\end{array}$ \\
\hline 0 & $\begin{array}{l}10.05 \\
(2.02)\end{array}$ & $\mathrm{a}$ & $\begin{array}{c}9.28 \\
(1.87)\end{array}$ & $\mathrm{a}$ & $\begin{array}{c}6.83 \\
(1.90)\end{array}$ & $\mathrm{a}$ & $\begin{array}{c}11.37 \\
(2.10)\end{array}$ & $\mathrm{a}$ \\
\hline 12 & $\begin{array}{l}15.84 \\
(1.58)\end{array}$ & $\mathrm{b}$ & $\begin{array}{l}13.07 \\
(1.63)\end{array}$ & $\mathrm{b}$ & $\begin{array}{c}8.67 \\
(2.06)\end{array}$ & $a b$ & $\begin{array}{l}16.28 \\
(1.92)\end{array}$ & $a b$ \\
\hline 24 & $\begin{array}{l}16.88 \\
(1.60)\end{array}$ & $\mathrm{b}$ & $\begin{array}{l}14.98 \\
(1.61)\end{array}$ & $\mathrm{bc}$ & $\begin{array}{l}11.42 \\
(1.96)\end{array}$ & bc & $\begin{array}{l}16.86 \\
(1.78)\end{array}$ & $\mathrm{b}$ \\
\hline 36 & $\begin{array}{l}19.95 \\
(0.97)\end{array}$ & $\mathrm{c}$ & $\begin{array}{l}17.04 \\
(1.34)\end{array}$ & $\mathrm{c}$ & $\begin{array}{l}15.26 \\
(1.80)\end{array}$ & $\mathrm{c}$ & $\begin{array}{c}21.17 \\
(1.05)\end{array}$ & $\mathrm{c}$ \\
\hline 48 & $\begin{array}{l}19.99 \\
(0.94)\end{array}$ & $\mathrm{c}$ & $\begin{array}{l}17.46 \\
(1.15)\end{array}$ & $\mathrm{c}$ & $\begin{array}{c}13.62 \\
(1.94)\end{array}$ & $\mathrm{c}$ & $\begin{array}{c}20.93 \\
(1.00)\end{array}$ & $\mathrm{c}$ \\
\hline
\end{tabular}

${ }^{a-c}$ Means within a column with different letters differ significantly $(P<0.05)$.

${ }^{1}$ Bedding samples taken at $0 \mathrm{~h}$, and $12,24,36$, and $48 \mathrm{~h}$ after treatments were applied.

${ }^{2}$ Standard errors for post-ANOVA contrasts.

Table 4. Sample mean (ln-transformed cfu/g of DM) and post-ANOVA groups for bacterial counts from bedding by stall area over all samplings

\begin{tabular}{|c|c|c|c|c|c|c|c|c|}
\hline \multirow[b]{2}{*}{ Stall area ${ }^{1}$} & \multicolumn{2}{|c|}{ Coliforms } & \multicolumn{2}{|c|}{ Klebsiella spp. } & \multicolumn{2}{|c|}{ Escherichia coli } & \multicolumn{2}{|c|}{ Streptococcus spp. } \\
\hline & $\begin{array}{c}\text { Mean } \\
(\mathrm{SE})\end{array}$ & $\begin{array}{c}\text { Contrast }^{2} \\
(1.03)\end{array}$ & $\begin{array}{c}\text { Mean } \\
(\mathrm{SE})\end{array}$ & $\begin{array}{c}\text { Contrast } \\
(1.26)\end{array}$ & $\begin{array}{c}\text { Mean } \\
(\mathrm{SE})\end{array}$ & $\begin{array}{c}\text { Contrast } \\
\text { (NA) }\end{array}$ & $\begin{array}{c}\text { Mean } \\
(\mathrm{SE})\end{array}$ & $\begin{array}{c}\text { Contrast } \\
(1.20)\end{array}$ \\
\hline I & $\begin{array}{l}17.45 \\
(1.68)\end{array}$ & $\mathrm{a}$ & $\begin{array}{l}16.00 \\
(1.55)\end{array}$ & a & $\begin{array}{c}13.32 \\
(2.08)\end{array}$ & $\mathrm{a}$ & $\begin{array}{c}17.98 \\
(1.64)\end{array}$ & $a b$ \\
\hline II & $\begin{array}{l}17.75 \\
(1.47)\end{array}$ & $\mathrm{a}$ & $\begin{array}{l}15.71 \\
(1.48)\end{array}$ & $\mathrm{a}$ & $\begin{array}{l}12.34 \\
(1.97)\end{array}$ & $\mathrm{a}$ & $\begin{array}{c}17.51 \\
(1.60)\end{array}$ & $a b$ \\
\hline III & $\begin{array}{l}15.09 \\
(1.79)\end{array}$ & $\mathrm{b}$ & $\begin{array}{l}11.74 \\
(1.84)\end{array}$ & $\mathrm{b}$ & $\begin{array}{l}10.37 \\
(1.92)\end{array}$ & $\mathrm{a}$ & $\begin{array}{l}16.26 \\
(1.98)\end{array}$ & a \\
\hline IV & $\begin{array}{l}18.37 \\
(1.23)\end{array}$ & a & $\begin{array}{l}16.40 \\
(1.34)\end{array}$ & $\mathrm{a}$ & $\begin{array}{l}11.00 \\
(2.02)\end{array}$ & a & $\begin{array}{l}19.24 \\
(1.52)\end{array}$ & $\mathrm{b}$ \\
\hline V & $\begin{array}{l}14.06 \\
(1.86)\end{array}$ & $\mathrm{b}$ & $\begin{array}{l}12.00 \\
(1.734)\end{array}$ & $\mathrm{b}$ & $\begin{array}{c}8.77 \\
(2.05)\end{array}$ & a & $\begin{array}{c}15.61 \\
(2.06)\end{array}$ & $\mathrm{a}$ \\
\hline
\end{tabular}

${ }^{\mathrm{a}, \mathrm{b}}$ Means within a column with different letters differ $(P<0.05)$.

${ }^{1}$ Five groups of 30 -cow free-stall areas.

${ }^{2}$ Standard errors for post-ANOVA contrasts. 
Table 5. Sample mean (ln-transformed cfu) and post-ANOVA groups for bacterial counts from teat end samples by treatment group over all samplings

\begin{tabular}{|c|c|c|c|c|c|c|c|c|}
\hline \multirow[b]{2}{*}{ Treatment $^{1}$} & \multicolumn{2}{|c|}{ Coliforms } & \multicolumn{2}{|c|}{ Klebsiella spp. } & \multicolumn{2}{|c|}{ Escherichia coli } & \multicolumn{2}{|c|}{ Streptococcus spp. } \\
\hline & $\begin{array}{c}\text { Mean } \\
(\mathrm{SE})\end{array}$ & $\begin{array}{c}\text { Contrast }^{2} \\
(0.87)\end{array}$ & $\begin{array}{c}\text { Mean } \\
(\mathrm{SE})\end{array}$ & $\begin{array}{c}\text { Contrast } \\
(1.04)\end{array}$ & $\begin{array}{c}\text { Mean } \\
(\mathrm{SE})\end{array}$ & $\begin{array}{c}\text { Contrast } \\
(1.18)\end{array}$ & $\begin{array}{c}\text { Mean } \\
(\mathrm{SE})\end{array}$ & $\begin{array}{c}\text { Contrast } \\
(1.03)\end{array}$ \\
\hline Lime & $\begin{array}{l}15.29 \\
(1.01)\end{array}$ & $\mathrm{a}$ & $\begin{array}{l}12.69 \\
(1.38)\end{array}$ & $\mathrm{a}$ & $\begin{array}{l}10.15 \\
(1.33)\end{array}$ & $a b$ & $\begin{array}{l}13.36 \\
(0.97)\end{array}$ & $\mathrm{a}$ \\
\hline Shavings & $\begin{array}{l}16.54 \\
(0.97)\end{array}$ & $a b$ & $\begin{array}{l}15.17 \\
(0.97)\end{array}$ & $\mathrm{b}$ & $\begin{array}{c}9.22 \\
(1.56)\end{array}$ & $\mathrm{a}$ & $\begin{array}{c}13.31 \\
(0.93)\end{array}$ & a \\
\hline Fly ash & $\begin{array}{c}17.61 \\
(0.89)\end{array}$ & $\mathrm{b}$ & $\begin{array}{l}15.71 \\
(0.82)\end{array}$ & $\mathrm{b}$ & $\begin{array}{l}13.65 \\
(1.28)\end{array}$ & $\mathrm{c}$ & $\begin{array}{c}15.42 \\
(0.84)\end{array}$ & a \\
\hline CAC & $\begin{array}{l}17.73 \\
(0.80)\end{array}$ & $\mathrm{b}$ & $\begin{array}{l}16.19 \\
(0.80)\end{array}$ & $\mathrm{b}$ & $\begin{array}{l}12.09 \\
(1.44)\end{array}$ & bc & $\begin{array}{l}14.10 \\
(1.03)\end{array}$ & $\mathrm{a}$ \\
\hline Control & $\begin{array}{c}17.92 \\
(0.67)\end{array}$ & $\mathrm{b}$ & $\begin{array}{l}16.29 \\
(0.72)\end{array}$ & $\mathrm{b}$ & $\begin{array}{l}11.57 \\
(1.48)\end{array}$ & abc & $\begin{array}{c}14.98 \\
(0.83)\end{array}$ & $\mathrm{a}$ \\
\hline
\end{tabular}

${ }^{\mathrm{a}-c}$ Means within a column with different letters differ significantly $(P<0.05)$.

${ }^{1}$ Treatments: lime $=0.5 \mathrm{~kg}$ of hydrated lime; shavings $=1 \mathrm{~kg}$ of kiln-dried wood shavings; fly ash $=1 \mathrm{~kg}$ of coal fly ash; $\mathrm{CAC}=$ commercial acidic conditioner $(300 \mathrm{~mL}$ for first and $120 \mathrm{~mL}$ for subsequent applications in each cycle); control = no bedding.

${ }^{2}$ Standard errors for post-ANOVA contrasts.

spp. compared with the control (Table 5). The other treatments (CAC, fly ash, and shavings) did not differ from the control or from each other in terms of bacterial counts for coliforms, Klebsiella spp., or Streptococcus spp. The counts of $E$. coli appeared to be erratic (Table 5).

Similar to the mattress sample results, there was a significant effect of sampling time on bacterial counts of teat swab samples $(P<0.0006)$. As indicated in Table 6 , bacterial populations were lowest at $0 \mathrm{~h}$ for all 4 types of bacteria. However, unlike the mattress samples, which showed a trend of increase from 12 to $48 \mathrm{~h}$ for all bacterial types (Table 3), there was no difference among the 4 sampling times $(12,24,36$, and $48 \mathrm{~h})$ in coliform or Streptococcus spp. counts associated with the teat swabs. For Klebsiella spp. and E. coli, there were small but sometimes significant differences during 12, 24, 36, and $48 \mathrm{~h}$ (Table 6).

Stall area appeared to have significant impact on the mean natural log-transformed populations of coliforms $(P<0.028)$, but not Klebsiella spp., E. coli, or Streptococcus spp. Coliform counts were lower in stall area $\mathrm{V}$ compared with stall areas III and IV (Table 7).

\section{DISCUSSION}

\section{Mattresses}

Hydrated lime has been shown to effectively lower bacterial counts when added to organic bedding materials. For example, the addition of hydrated lime to sawdust significantly reduced bacterial counts for $1 \mathrm{~d}$ in a

Table 6. Sample mean (ln-transformed cfu) and post-ANOVA groups for bacterial counts from all teat-end samples by sampling time

\begin{tabular}{|c|c|c|c|c|c|c|c|c|}
\hline \multirow[b]{2}{*}{ Sample time, ${ }^{1} \mathrm{~h}$} & \multicolumn{2}{|c|}{ Coliforms } & \multicolumn{2}{|c|}{ Klebsiella spp. } & \multicolumn{2}{|c|}{ Escherichia coli } & \multicolumn{2}{|c|}{ Streptococcus spp. } \\
\hline & $\begin{array}{c}\text { Mean } \\
(\mathrm{SE})\end{array}$ & $\begin{array}{c}\text { Contrast }^{2} \\
(0.87)\end{array}$ & $\begin{array}{c}\text { Mean } \\
(\mathrm{SE})\end{array}$ & $\begin{array}{c}\text { Contrast } \\
(1.04)\end{array}$ & $\begin{array}{c}\text { Mean } \\
(\mathrm{SE})\end{array}$ & $\begin{array}{c}\text { Contrast } \\
(1.18)\end{array}$ & $\begin{array}{c}\text { Mean } \\
(\mathrm{SE})\end{array}$ & $\begin{array}{c}\text { Contrast } \\
(1.03)\end{array}$ \\
\hline 0 & $\begin{array}{c}12.91 \\
(0.85)\end{array}$ & $\mathrm{a}$ & $\begin{array}{c}10.37 \\
(1.02)\end{array}$ & a & $\begin{array}{c}8.26 \\
(1.21)\end{array}$ & $\mathrm{a}$ & $\begin{array}{c}7.84 \\
(1.23)\end{array}$ & a \\
\hline 12 & $\begin{array}{l}18.64 \\
(1.13)\end{array}$ & $\mathrm{b}$ & $\begin{array}{l}17.09 \\
(1.06)\end{array}$ & bc & $\begin{array}{l}12.01 \\
(1.73)\end{array}$ & $\mathrm{bc}$ & $\begin{array}{c}16.37 \\
(0.37)\end{array}$ & $\mathrm{b}$ \\
\hline 24 & $\begin{array}{l}17.41 \\
(0.61)\end{array}$ & $\mathrm{b}$ & $\begin{array}{l}16.11 \\
(0.59)\end{array}$ & bc & $\begin{array}{l}11.58 \\
(1.31)\end{array}$ & $\mathrm{bc}$ & $\begin{array}{c}14.54 \\
(0.36)\end{array}$ & $\mathrm{b}$ \\
\hline 36 & $\begin{array}{l}18.74 \\
(0.58)\end{array}$ & $\mathrm{b}$ & $\begin{array}{l}17.46 \\
(0.61)\end{array}$ & $\mathrm{b}$ & $\begin{array}{l}13.74 \\
(1.35)\end{array}$ & $\mathrm{b}$ & $\begin{array}{c}16.37 \\
(0.42)\end{array}$ & $\mathrm{b}$ \\
\hline 48 & $\begin{array}{c}17.39 \\
(0.61)\end{array}$ & $\mathrm{b}$ & $\begin{array}{c}15.03 \\
(0.89)\end{array}$ & c & $\begin{array}{l}11.08 \\
(1.39)\end{array}$ & c & $\begin{array}{c}16.04 \\
(0.45)\end{array}$ & $\mathrm{b}$ \\
\hline
\end{tabular}

${ }^{\mathrm{a}-\mathrm{c}}$ Means within a column with different letters differ significantly $(P<0.05)$.

${ }^{1}$ Teat-end bacterial samples taken at $0 \mathrm{~h}$, and $12,24,36$, and $48 \mathrm{~h}$ after treatments were applied.

${ }^{2}$ Standard errors for post-ANOVA contrasts. 
Table 7. Sample mean (ln-transformed cfu) and post-ANOVA groups for bacterial counts from teat ends by stall area over all samplings

\begin{tabular}{|c|c|c|}
\hline \multirow[b]{2}{*}{$\begin{array}{l}\text { Stall } \\
\text { area }^{1}\end{array}$} & \multicolumn{2}{|c|}{ Coliforms } \\
\hline & $\begin{array}{l}\text { Mean } \\
\text { (SE) }\end{array}$ & $\begin{array}{c}\text { Contrast }^{2} \\
(0.87)\end{array}$ \\
\hline I & $\begin{array}{c}17.02 \\
(0.81)\end{array}$ & $a b$ \\
\hline II & $\begin{array}{l}16.80 \\
(0.84)\end{array}$ & $a b$ \\
\hline III & $\begin{array}{l}17.67 \\
(0.67)\end{array}$ & $\mathrm{a}$ \\
\hline IV & $\begin{array}{c}18.19 \\
(0.87)\end{array}$ & $\mathrm{a}$ \\
\hline V & $\begin{array}{c}15.42 \\
(1.13)\end{array}$ & $\mathrm{b}$ \\
\hline
\end{tabular}

\footnotetext{
${ }^{\mathrm{a}, \mathrm{b}}$ Means within a column with different letters differ significantly $(P<0.05)$.

${ }^{1}$ Five groups of 30 -cow free-stall areas.

${ }^{2}$ Standard error for post-ANOVA contrast.
}

tie-stall study (Hogan and Smith, 1997), whereas in a second study (also tie-stalls), no statistical differences were detected although bacterial counts were numerically lower in lime-treated sawdust than in the untreated sawdust control (Hogan et al., 1999).

For the first time, the present study showed that hydrated lime can effectively reduce bacterial growth on free-stall mattresses when used as the sole bedding treatment. Compared with the other treatments, the hydrated lime treatment produced the lowest bacterial counts of all bacterial types. However, the amount of hydrated lime used in the present study $(0.5 \mathrm{~kg}$ per stall every $48 \mathrm{~h}$ ) did cause some irritation to the skin of the udder and legs. Approximately one-third of the cows in the hydrated lime treatment group had evidence of mild ulceration and scaling on both the legs and udder. The lesions were localized to areas where the lime had caked on the skin caused by the mixture of the hydrated lime with manure, milk, or urine. Lesions were evident approximately $3 \mathrm{~d}$ after exposure to the hydrated lime. This suggests that routine long-term use of more than $0.5 \mathrm{~kg}$ of lime alone on mattresses may be associated with undesirable side effects despite the benefit in bacteria suppression. The extent of irritation caused by hydrated lime when used as the sole bedding on mattresses could be exacerbated by stall designs that allow more manure to be deposited on the back of the mattress. Further studies may be warranted to test the efficacy of lower application rates to avoid the skin irritation problem.

The CAC was applied according to the manufacturer's recommendation and did not cause skin irritation or any other visible problems. The commercial product contains $92.5 \%$ calcium carbonate, $5 \%$ sodium dichloroisocyanurate, and $2.5 \%$ aluminum sulfate and has a $\mathrm{pH}$ of 3.7. The ingredient sodium dichloroisocyanurate has been used as part of a teat dip formulation to control mastitis in dairy cows (Boddie and Nickerson, 1996) and as a hospital disinfectant (Bloomfield and Uso, 1985). In the present study, CAC was effective in suppressing the populations of coliforms, Klebsiella spp., and Streptococcus spp. although its efficacy was less than that of hydrated lime. It is not clear whether the antibacterial feature was mainly due to chlorine generated by the sodium dichloroisocyanurate or a combined effect with the low $\mathrm{pH}$.

The fly ash treatment resulted in lower coliform counts than shavings or the control and in lower Klebsiella spp. counts than shavings and could serve as an alternative to shavings on mattresses. In a previous study, fly ash obtained from a different source showed antibacterial effects on the first day when mixed with sawdust and used on tie-stall mattresses (Z. Dou, unpublished data). University of California researchers claimed that adding fly ash to manure solids used for bedding was beneficial in reducing the bacterial load (Brazil et al., 1999), but they cautioned that fly ash characteristics should be evaluated before using it as a bedding source because fly ash from different sources had different $\mathrm{pH}$ values and its effectiveness in suppressing bacterial growth is related to $\mathrm{pH}$. The fly ash material used in the present study had a $\mathrm{pH}$ of 11.4, whereas other fly ash materials could have $\mathrm{pH}$ values as low as 7.9 or as high as 13 (Dou et al., 2003). It is not clear if there is a $\mathrm{pH}$ threshold regarding fly ash's efficacy in suppressing bacterial growth. On the other hand, dust, which may affect the cows' breathing, appeared to be a nuisance when using fly ash as the sole bedding material in this study. Fly ash did not appear to cause any skin irritation.

Notably, the use of shavings resulted in the highest Klebsiella spp. counts compared with all bedding materials, although the difference between shavings and control was not statistically significant (Table 2). Outbreaks of Klebsiella spp. mastitis have been attributed to sawdust and shavings contaminated with Klebsiella spp. (Newman and Kowalski, 1973). In the present study, shavings had lower Streptococcus spp. counts but comparable counts for the other bacterial groups compared with the no-bedding control.

Time zero was defined as the beginning of the sampling period when cows had not had access to freshly bedded stalls. The lowest bacterial counts were found at $0 \mathrm{~h}$ for coliforms and Klebsiella spp. For Steptococcus spp. and $E$. coli, counts at $0 \mathrm{~h}$ were lower than at 24 , 36, and $48 \mathrm{~h}$. Also, coliform and Streptococcus spp. counts were higher at 36 and $48 \mathrm{~h}$ than at 12 and 24 h, whereas Klebsiella spp. and E. coli counts were higher at 36 and $48 \mathrm{~h}$ than at $12 \mathrm{~h}$. A general trend 
was that bacterial numbers on the mattresses increased over time and were highest at 36 and $48 \mathrm{~h}$, suggesting that daily bedding would be superior to alternate-day bedding for controlling bacteria on mattresses at this dairy. Presumably cows would be exposed to fewer bacteria for a longer period when new bedding was added daily.

\section{Teats}

The only notable treatment effect on teat-end bacteria was associated with the hydrated lime treatment, which reduced both coliform and Klebsiella spp. counts. For E. coli and Streptococcus spp., the teat-end samples with the lime treatment were similar to the other treatments in most cases. Comparing bacterial counts of the mattress samples (Table 2) with those of the teat swabs (Table 5), it seemed that much of the treatment effect on suppressing bacterial growth on the mattresses diminished in the teat samples. In other words, suppressed bacterial growth on the mattresses did not always transfer to lower bacterial numbers on the teat ends. Teat ends can be in contact with manure or other surfaces in addition to mattresses. As pointed out by Rendos et al. (1975), bacterial populations on teat ends are greatly influenced by uncontrolled environmental factors.

It is not surprising that all bacterial counts for teatend samples were consistently the lowest at $0 \mathrm{~h}$ compared with other times, because the 0 -h samples were taken after the cows were milked and their teats were postdipped, whereas all other samples were taken before the cow's teats were predipped upon entrance into the parlor. For the subsequent sampling times, the small differences in bacterial counts between the first day (12 to $24 \mathrm{~h}$ ) and second day (36 to $48 \mathrm{~h}$ ) shown in mattress samples diminished with the teat-end samples (Tables 3 and 6 ), because bacterial counts were similarly high from 12 to $48 \mathrm{~h}$. We presume that the lack of a treatment effect over time on teat-end bacterial counts was a result of bacteria growth prevailing over the weak treatment effect. It is unlikely that differences in storage time for samples collected at various times $(0,12,24,36$, and $48 \mathrm{~h})$ would have had considerable impact on the results considering the findings from the preliminary trial. We do not know why Klebsiella spp. and $E$. coli counts on teat ends were lower at $48 \mathrm{~h}$ than at $36 \mathrm{~h}$. Previous studies have found a modest correlation between bacterial counts on the teat ends and in sawdust bedding (Rendos et al., 1975; Hogan et al., 1999; Zdanowicz et al., 2004), whereas Zdanowicz et al. (2004) reported a weak correlation between bacterial counts in sand bedding and on teat ends.
In summary, results from the present study suggest that hydrated lime was the only treatment that significantly reduced bacterial counts of coliforms, Klebsiella spp., E. coli, and Streptococcus spp. on mattress samples. Hydrated lime also reduced bacterial counts of both coliforms and Klebsiella spp. in teat-end samples. Although fly ash and CAC effectively suppressed some bacterial groups on the mattresses compared with the no-bedding control or shavings, they had little effect on reducing bacterial counts at the teat ends. None of the treatments was effective in reducing Streptococcus spp. counts on the teats. Additional work is needed regarding the quantity and frequency of application of hydrated lime used as the sole bedding material on freestall mattresses to minimize skin irritation and maximize its antibacterial efficacy. Further research is needed to develop economical, non-dust-producing, and nonirritating products with antibacterial effects that effectively reduce the numbers of bacteria on mattresses and teat ends in free-stall conditions, where cows are repeatedly contaminating and removing bedding with their feet as they enter and exit the freestalls. In addition, identification of current and novel management practices that reduce the contamination of manure on teats between milking times in free-stalls is needed.

\section{ACKNOWLEDGMENTS}

The authors thank the personnel at University of Pennsylvania's Marshak Dairy for their assistance with this study. Thanks also go to Charles Benson, Sheri Young, and Lynn Stephens for their laboratory support.

\section{REFERENCES}

Bloomfield, S. F., and E. E. Uso. 1985. The antibacterial properties of sodium hypochlorite and sodium dichloroisocyanurate as hospital disinfectants. J. Hosp. Infect. 6:20-30.

Boddie, R. L., and S. C. Nickerson. 1996. Efficacy of teat dips containing a hypochlorous acid germicide against experimental challenge with Staphylococcus aureus and Streptococcus agalactiae. J. Dairy Sci. 79:1683-1688.

Brazil, L., J. Kirk, R. Finger, C. Holmberg, W. Sischo, and G. Higginbotham. 1999. Studies on the potential use of fly ash in bedding on dairies. http://www.vetmed.ucdavis.edu/vetext/INF-DA/INFDA_Flyash99.html Accessed June 27, 2007.

DeGraves, F. J., and J. Fetrow. 1993. Economics of mastitis and mastitis control. Vet. Clin. North Am. Food Anim. Pract. 9:421-434.

Dou, Z., G. Y. Zhang, W. L. Stout, J. D. Toth, and J. D. Ferguson. 2003. Efficacy of alum and coal combustion by-products in stabilizing manure phosphorus. J. Environ. Qual. 32:1490-1497.

Hogan, J. S., V. L. Bogacz, L. M. Thompson, S. Romig, P. S. Schoenberger, W. P. Weiss, and K. L. Smith. 1999. Bacterial counts associated with sawdust and recycled manure bedding treated with commercial conditioners. J. Dairy Sci. 82:1690-1695.

Hogan, J. S., and K. L. Smith. 1997. Bacteria counts in sawdust bedding. J. Dairy Sci. 80:1600-1605.

Hogan, J. S., K. L. Smith, K. H. Hoblet, D. A. Todhunter, P. S. Schoenberger, W. D. Hueston, D. E. Pritchard, G. L. Bowman, 
L. E. Heider, B. L. Brockett, and H. R. Conrad. 1989. Bacterial counts in bedding materials used on nine commercial dairies. J. Dairy Sci. 72:250-258.

Hogan, J. S., S. L. Wolf, and C. S. Petersson-Wolfe. 2007. Bacterial counts in organic materials used as free-stall bedding following treatment with commercial conditioner. J. Dairy Sci. 90:10581062.

Natzke, R. P., and B. J. LeClair. 1976. Coliform contaminated bedding and new infections. J. Dairy Sci. 59:2152-2154.

Newman, L. E., and J. J. Kowalski. 1973. Fresh sawdust beddingA possible source of Klebsiella organisms. Am. J. Vet. Res. 34:979-980.
Rendos, J. J., R. J. Eberhart, and E. M. Kesler. 1975. Microbial populations on teat ends of dairy cows and bedding materials. J. Dairy Sci. 58:1492-1500.

Smith, K. L., and J. S. Hogan. 2001. The World of Mastitis. Pages 112 in Proc. 2nd Int. Symp. Mastitis and Milk Quality, Vancouver, Canada. Natl. Mastitis Counc., Madison, WI, and Am. Assoc. Bovine Pract., Rome, GA.

Zdanowicz, M., J. A. Shelford, C. B. Tucker, D. M. Weary, and M. A. G. von Keyserlingk. 2004. Bacterial populations on teat ends of dairy cows housed in free stalls and bedded with either sand or sawdust. J. Dairy Sci. 87:1694-1701. 